\title{
Effectiveness of a WeChat Combined Continuous Flash Glucose Monitoring System on Glycemic Control in Juvenile Type I Diabetes Mellitus Management: Randomized Controlled Trial
}

This article was published in the following Dove Press journal:

Diabetes, Metabolic Syndrome and Obesity: Targets and Therapy

Yuejie Xu, (D) ${ }^{1}, *$ Lei $\mathrm{Xu},{ }^{1, *}$ Weijing Zhao,' Qing Li, Ming Li,' Wei Lu,' Hui Zeng,' Jinhua Yan, ${ }^{2}$ Daizhi Yang, ${ }^{2}$ Wei Wu, ${ }^{3}$ jianping Weng, ${ }^{2}$ Jiemin Pan,' Fang Liu'

'Department of Endocrinology and Metabolism, Shanghai Jiao Tong University Affiliated Sixth People's Hospital, Shanghai Clinical Center for Diabetes, Shanghai Key Clinical Center for Metabolic Disease, Shanghai Diabetes Institute, Shanghai Key Laboratory of Diabetes Mellitus, Shanghai, 200233, People's Republic of China; ${ }^{2}$ Department of Endocrinology and Metabolic Disease, The Third Affiliated Hospital of Sun Yat-Sen University, Guangzhou, 510630, People's Republic of China; ${ }^{3}$ Department of Pediatrics, Tongii Hospital, Wuhan Tongji Medical University, Wuhan, Hubei, 430030, People's Republic of China

*These authors contributed equally to this work

Correspondence: Jiemin Pan; Fang Liu Department of Endocrinology and Metabolism, Shanghai Jiao Tong University Affiliated Sixth People's Hospital, Shanghai Clinical Center for Diabetes, Shanghai Key Clinical Center for Metabolic Disease, Shanghai Diabetes Institute, Shanghai Key Laboratory of Diabetes Mellitus, 600 Yishan Road, Xuhui District, Shanghai, 200233, People's Republic of China

Tel +86-|8930I73803

Email lilypjm@hotmail.com;

f-liu@sjtu.edu.cn
Purpose: Smartphones have received increasing attention and achieved positive outcomes in diabetes intervention. The widespread use of WeChat in China provides an opportunity for self-management practices in patients with diabetes. Nevertheless, how to combine the strengths of the WeChat platform with traditional medical strategy remains to be explored. This study aimed to evaluate the efficacy of a novel flash glucose monitoring device combined with the WeChat platform in juvenile type 1 diabetes management.

Patients and Methods: A total of 60 juvenile patients with type 1 diabetes were randomly assigned into three groups: a blood glucose self-monitoring group (group A), a flash glucose monitoring (group B), and a flash glucose monitoring combined WeChat-interactive management group (group C). The intergroup differences in demographics, biochemical indicators, and questionnaire scores of the Diabetes Monitoring and Treatment Satisfaction Questionnaire and Diabetes Specific Quality of Life assessment were compared at the baseline and after 6 months. Results: After the 6-month intervention, groups $\mathrm{B}$ and $\mathrm{C}$ showed significantly lower glycated hemoglobin A1c (HbA1c) levels compared to those observed at baseline (both $P<0.05$ ), with the largest decrease observed in group $\mathrm{C}$ (group $\mathrm{B}$ vs group $\mathrm{C}, P=0.04$ ). Hypoglycemic episodes per month decreased from baseline in groups B and C (both $\mathrm{P}<0.05$ ) and were more significant in group $\mathrm{C}(P<0.001)$. In addition, the DMTSQ scores increased in the 6th month in all groups (all $P<0.05$ ), and the largest rise in scores was found in group $\mathrm{C}$, followed by groups $\mathrm{B}$ and $\mathrm{A}$. The DQOL scores in groups B and C decreased significantly from the baseline (both $P<0.05$ ), with no change in group A.

Conclusion: Flash glucose monitoring combined with the WeChat-interactive system may help achieve sustained glycemic control and higher satisfaction in patients with juvenile type 1 diabetes.

Trial Registration: This study was registered at chictr.org.cn, number ChiCTR1900025495. Registered 29 August 2019.

Keywords: WeChat, type 1 diabetes, self-management, glycated hemoglobin A1c

\section{Introduction}

Type 1 diabetes mellitus (T1D) is an autoimmune disease in which immune cells attack and destroy insulin-producing pancreatic $\beta$ cells. According to the latest nationwide population-based registry study, the estimated incidence of T1D per 100,000 person-years for all ages in China was $1.01 .^{1}$ 
Early glycemic control is particularly important for preventing the development and progression of chronic complications of diabetes. It is well known that hypoglycemia causes cognitive impairment and mood changes, such as depression, irritation, and fear. In severe cases, changes in brain metabolism can even cause permanent sequelae and be potentially life-threatening. ${ }^{2}$ The goal of diabetes treatment in adolescents is to maintain blood glucose level within the normal range without recurrent or severe hypoglycemia; that is, a balance between hyperglycemia treatment and minimizing the frequency of hypoglycemic episodes should be maintained. ${ }^{3}$

Regular self-monitoring of blood glucose (SMBG) is particularly important for patients with T1D to assess glycemic control, determine the risk of hypoglycemia, and respond promptly. ${ }^{4}$ However, it is also accompanied by the burden and pain associated with multiple needle pricks for blood collection and the complexity of the procedures for use. Continuous glucose monitoring systems (CGM) measure interstitial fluid glucose concentrations (ISFG) throughout the day and night; thus, they can provide much more sufficient blood glucose management information than SMBG. ${ }^{5,6}$ The newly developed Abbott FreeStyle Libre is a flash glucose monitoring system (FGM) that requires a reader to scan the subcutaneous probe to obtain glycemic values.

$\mathrm{HbAlc}$ is currently recognized as the key surrogate marker for developing long-term diabetes complications and has been used as the primary endpoint for many CGM studies. HbA1c reflects average glucose over the last 2-3 months, but it does not reflect glycemic variability. The use of FreeStyle Libre allows not only recurrent painless glucose sampling but also a real-time observation of glycemic excursions and daily profiles, which can impact immediate therapy decisions.

Achieving optimal glycemic control requires intensive self-management for adolescents with T1D. However, adolescents with T1D struggle with achieving therapeutic targets for their shortage of medical knowledge, variable lifestyles, and less outpatient visits. Additionally, owing to many patients with diabetes and the shortage of medical staff in China, clinicians and nurses are often too overwhelmed to provide normative and continuous management for patients with diabetes. The widespread use of smartphones, especially among the younger population, offers an opportunity to provide diabetes information and interaction with physicians. ${ }^{7}$ Given adolescents' propensity for new technology, web-based interventions may provide a feasible communication with healthcare providers and motivate them to improve self-management.

Although interest in this technology increases, the clinical value of these web-based interventions remains unclear. $^{8}$ To date, most studies were performed in adults with type 2 diabetes, rarely among adolescents with T1D. Furthermore, few of the web-based intervention provided users with personalized education, feedback, or motivation, which are emphasized in current clinical guidelines for behavior change and comprehensive selfmanagement. ${ }^{9,10}$

In China, WeChat is the most popular social networking platform; it is a free app characterized by high convenience and accessibility. Through the app, complex information can be presented through video and graphics and provide a feasible way to spread health information to the public. Many studies have shown that WeChat has great potential in health intervention. ${ }^{11,12}$ However, to our knowledge, no well-designed studies have focused on a WeChat-based intervention for T1D in China.

Therefore, this study aims to clarify the effect of the WeChat intervention combined with FGM management on glycemic control, hypoglycemia incidence, and life quality in T1D patients.

\section{Patients and Methods}

\section{Trial Design}

A parallel, three-group, randomized controlled trial study was carried out for 6 months. Participants were recruited from the outpatient clinic of the Department of Endocrinology and Metabolism of Shanghai Jiao Tong University Affiliated Sixth People's Hospital from January 2019 to June 2019. By using a set of random numbers, the participants were randomly assigned into three groups in a 1:1:1 ratio: group A (SMBG group), group B (FGM group), and group C (FGM combined WeChat-interactive management group). This study was registered at chictr.org.cn, number ChiCTR1900025495.

\section{Study Participants}

The study inclusion criteria were as follows: 1) adolescent patients (age 10-19 years) diagnosed with T1D with the criteria established by WHO in 1999, with diabetes duration longer than 1 year; 2) glycosylated hemoglobin A1c concentration between $7 \%$ and $10 \% ; 3$ ) use of multiple daily insulin (MDI) and continuous subcutaneous insulin infusion (CSII) for at least 3 months, stable diabetes 
medication regimen for 3 months before study entry (change in insulin $\leq 20 \%$ ); 4) previous documentation of blood glucose level self-monitoring regularly for 2 months (at least three times per day) and willingness to continue for at least 6 months; 5) willingness to wear CGM; 6) ability to speak, read, and write Chinese; 7) ability to use WeChat or have a family member able to use the WeChat platform.

The exclusion criteria included the following: 1) the use of CGM 3 months before study entry; 2) having severe diabetic complications such as diabetic retinopathy and diabetic nephropathy; 3) having a known allergy to medical-grade adhesives or CGM and its affiliated components; 4) being pregnant or planning pregnancy; 5) recent severe diseases like myocardial infarction, stroke, psychiatric diseases (historical/recent), malignant tumor, kidney disease (defined as eGFR $<45$ ), dermatosis, decided by the investigator; 6) current participation in another investigational study (must have completed any previous studies at least 30 days before being enrolled in this study); 7) current abuse of illicit drugs, alcohol, or prescription drugs; 8) any condition that could impact the reliability of the HbA1c measurement (eg, hemoglobinopathy, hemolytic anemia, chronic liver disease), decided by the investigator.

The study was approved by the Ethics Committee of the Shanghai Jiao Tong University Affiliated Sixth People's Hospital and conformed to the provisions of the Declaration of Helsinki (2018-011). Written informed consent was obtained from all patients before starting this enrollment. And written informed parental consent was obtained from all patients under the age of 18 before starting this enrollment.

\section{Grouping and Intervention}

Patients were randomly assigned to groups $\mathrm{A}, \mathrm{B}$, or $\mathrm{C}$ of conventional blood glucose monitoring, flash glucose monitoring, and flash glucose monitoring combined with WeChat monitoring, respectively. We recorded the data on demographics, laboratory findings, and questionnaire results of the patients at baseline and at the 6-month follow-up.

In Group A, a conventional home glucometer was used to monitor blood glucose $\geq$ three times a day, and the blood glucose monitoring values were uploaded to the Wenjuan survey platform.

In Group B, the flash blood glucose monitoring system (Libre 1, Abbott Diabetes Care Inc., Alameda, CA) was used. A specialist applied the flash glucose monitor to the back of the upper arm through a simple disposable applicator: a thin wire (flexible probe) was subcutaneously implanted, and the sensor was fixed to the application site with an adhesive film. It recorded the blood glucose value at 15-minute intervals automatically, and the blood glucose value can be determined at any time from the display.

In Group C, patients with the Abbott FreeStyle Libre monitor were asked to subscribe to a WeChat Official Account named "KongTangTianDi," which disseminates scientific diabetes-related information once a week. Furthermore, the WeChat Official Account platform was also used for real-time patient-doctor interactions. A thirdparty health manager was involved in interactive management with patients through the platform. Further, a nurse who specialized in diabetes helped analyze, evaluate, and review the glycemic monitoring data.

According to the study purpose, the functions of the WeChat program included several modules: (1) real-time registration and update of the child's health records; (2) sharing of diabetes-related knowledge including information on healthy diet, proper exercise, school and travel notifications, home environment and lifestyle guidance, and other practical medical knowledge; (3) answering of frequently left messages through text, audio or video; (4) making a remote diagnosis as well as regular online consultations; (5) provision of self-emergency guidance (call for consultation in acute situations), such as the occurrence of hypoglycemia or ketoacidosis (Figure 1).

Basic diabetes education, including adequate exercise, diet control, and regular follow-up, was conducted to the whole population after enrollment. All participants could contact clinicians on phone calls during follow-up; those who had subscribed to the WeChat Official Account were highly encouraged to contact clinicians through the platform.

\section{Data Collection}

Data were collected at baseline and at the 6-month follow-up for all participants. The data collected included information on demographic characteristics, anthropometric measurements, biochemical indicators, and questionnaires. The age, sex, past medical history, and lifestyle behaviors were recorded. Body mass index (BMI) was calculated as weight divided by height squared $\left(\mathrm{kg} / \mathrm{m}^{2}\right)$. Biochemical indicators included fasting plasma glucose (FPG), HbA1c, and blood lipid profile. Venous blood was collected in the morning after a 10-hour overnight fast. For FPG, blood samples were 


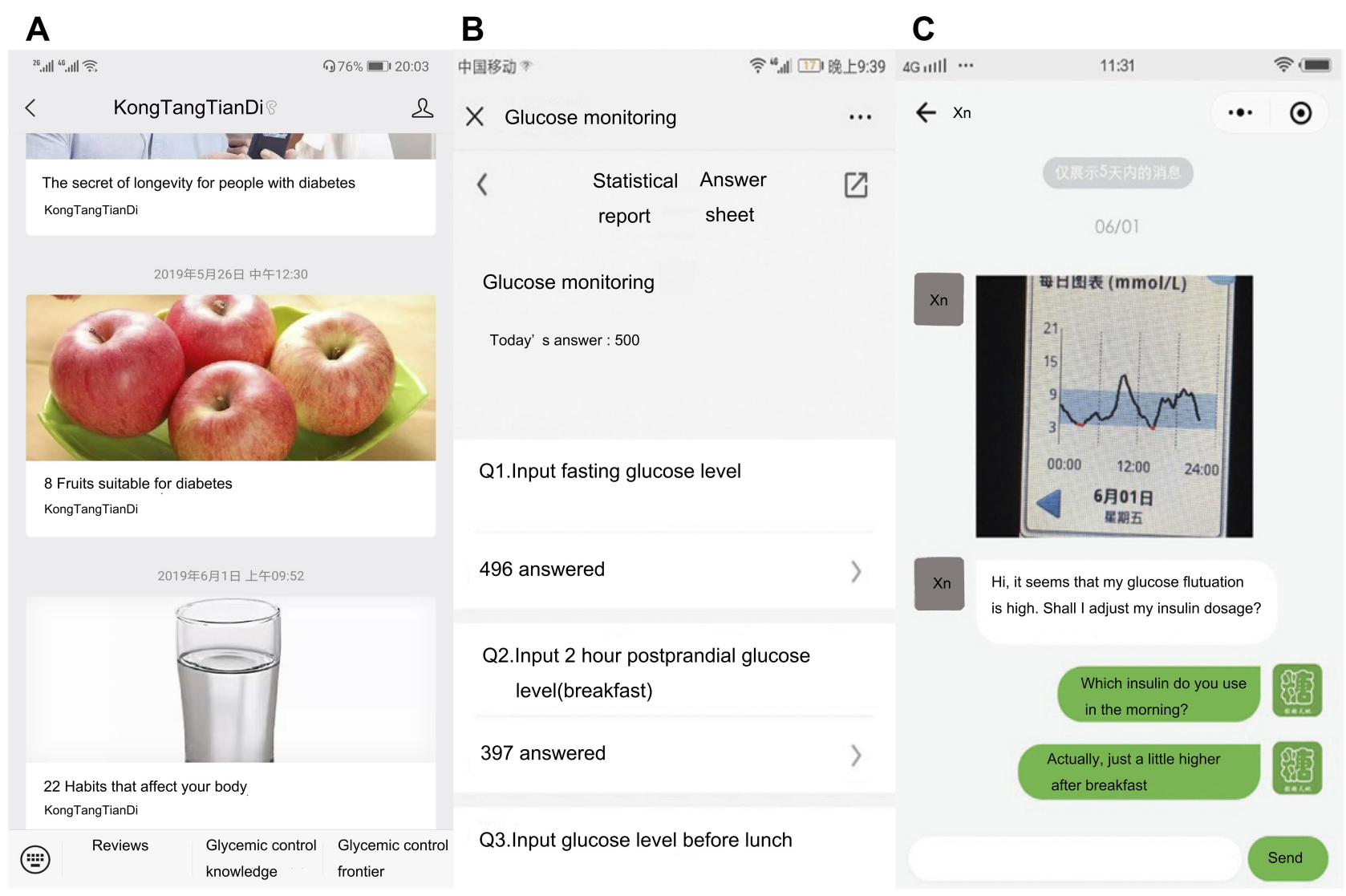

Figure I The screen captures to show how the KongTangTianDi WeChat public account works. (A) The platform module: diabetic frontier information and lifestyle guidance; (B) registration and update of the child's health records; (C) real-time online consultation.

collected into sodium fluoride + potassium oxalate anticoagulation tubes, and the plasma glucose was tested within 2 hours of sampling (glucose oxidase method; Shanghai Kehua Bio-Engineering Co., Ltd. kit, assayed on a Glamour2000 automated biochemical analyzer). The HbAlc (normal reference value: $4.3-6.5 \%$ ) was measured through highperformance liquid chromatography (Variant II hemoglobin testing system, Bio-Rad). The Hitachi 7080 automated biochemical analyzer was used for blood lipid panel testing. The questionnaires were divided into three parts and were completed with guidance from dedicated personnel. These were Diabetes Monitoring and Treatment Satisfaction Questionnaires (DMTSQ), Diabetes Quality of Life Measure (DQOL), and the Chinese Version Hypoglycemia Fear Survey II (CHFSII).

An episode of hypoglycemia is defined as glucose value by SMBG under $3.9 \mathrm{mmol} / \mathrm{L}$ or a presentation with classic symptoms. Hypoglycemic events in patients detected by Libre were recommended to retest fingertip blood glucose since the Libre is less accurate in low glucose levels.

\section{Sample Size}

The sample size was determined by estimating the change in $\mathrm{HbAlc}$ values assuming a $0.5 \%$ reduction in values in the intervention group. With alpha $=0.05$ and beta $=0.20$, we calculated an ideal sample size of 14 patients per group based on this preliminary study. After a dropout rate of $20 \%$ was incorporated, a sample size of more than 18 patients per group was required.

\section{Statistical Analysis}

All data were entered into SPSS [computer program] (Version 23.0. Armonk, NY: IBM Corp; 2015) by a specialist and analyzed. Data with a normal distribution were presented as mean and standard deviations (SD), and data with a non-normal distribution were presented as median with interquartile ranges (IQR). Analyses of variance (ANOVA) and covariance were used for intergroup comparisons of normally distributed data, whereas nonparametric analysis was used for non-normally distributed data. The Chi-Square test was used to compare 
proportions, and the linear correlation was used for single correlation analysis. Differences with $p<0.05$ (two-tailed) were considered statistically significant.

\section{Results}

\section{Baseline Characteristics}

Of the 120 participants who underwent screening in this study, 80 were enrolled and randomized to groups A $(n=30), B(n=25)$, or $C(n=25)$. Of these, 10 patients from group $\mathrm{A}, 5$ from group $\mathrm{B}$, and 5 from group $\mathrm{C}$ discontinued the study because they either withdrew their consent or were lost to follow-up (Figure 2). Thus, a total of 60 adolescent patients (31 males, 29 females; age, mean \pm SD $13.20 \pm 1.68$ years) with T1D were included, with 20 patients in each group (A, B, or
C). The general baseline characteristics, metabolic indicators, and questionnaire scores of the patients at the time of enrollment are shown in Table 1; no significant inter-group difference at baseline was observed.

\section{Changes in Glycemic Control}

After 6 months of study intervention, the HbA1c levels of Group A had no significant difference from the baseline $(7.75 \% \pm 1.34 \%$ vs $7.82 \% \pm 1.54 \%, p=0.30)$. While Group B had slightly declined $(7.2 \% \pm 1.88 \%$ vs $7.43 \% \pm 2.21 \%$, $p=0.04)$, and Group C decreased obviously $(7.26 \% \pm$ $0.89 \%$ vs $7.78 \% \pm 1.23 \%, p<0.001)$. The largest decrease was observed in Group C (Group B vs Group C, $p=0.04$ ). (Figure 3).

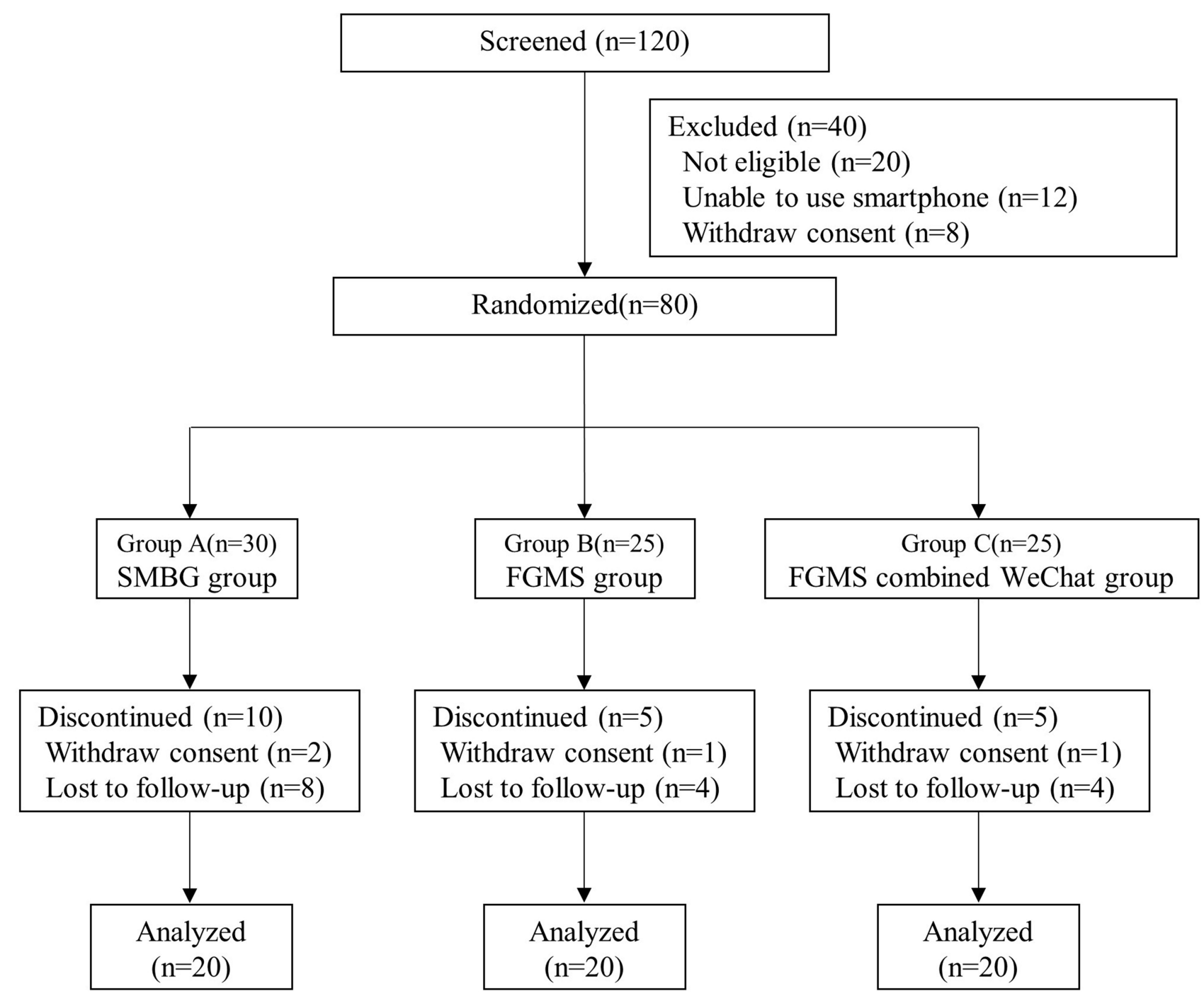

Figure 2 Study design and flow chart of participants recruitment and follow-up. 
Table I Clinical Characteristics and Questionnaire Scores of Three Groups at Baseline

\begin{tabular}{|l|c|c|c|}
\hline & Group A & Group B & Group C \\
\hline Age (years) & $13.60(1.27)$ & $13.35(1.90)$ & $12.65(1.73)$ \\
Gender (male/female) & $13 / 7$ & $11 / 9$ & $7 / 13$ \\
BMI (kg/m²) & $20.83(1.7 I)$ & $20.25(2.10)$ & $20.01(2.42)$ \\
Diabetes duration & $3.33(2.46)$ & $2.11(1.82)$ & $2.42(1.75)$ \\
(years) & & & \\
\hline Blood pressure (mmHg) & & & \\
Systolic & $117.00(10.14)$ & $111.88(9.75)$ & $109.79(11.15)$ \\
Diastolic & $69.06(6.76)$ & $69.35(6.75)$ & $68.05(9.47)$ \\
\hline Total cholesterol(mmol/L) & $4.86(0.85)$ & $4.58(0.89)$ & $4.80(0.92)$ \\
Triglyceride(mmol/L) & $0.75(0.46)$ & $1.00(1.12)$ & $0.71(0.24)$ \\
LDL-C(mmol/L) & $2.76(0.83)$ & $2.50(0.63)$ & $2.41(0.69)$ \\
HDL-C(mmol/L) & $1.44(0.31)$ & $1.52(0.54)$ & $1.76(0.4 I)$ \\
Fasting C peptide(ng/mL) & $0.62(0.70)$ & $0.57(0.67)$ & $0.42(0.46)$ \\
TIR (\%) & - & $61.60(20.40)$ & $63.35(17.54)$ \\
TAR (\%) & - & $27.15(21.46)$ & $25.70(18.30)$ \\
TBR (\%) & - & $11.25(11.74)$ & $10.95(11.05)$ \\
HbAIc (\%) & $7.82(1.54)$ & $7.43(2.21)$ & $7.78(1.23)$ \\
Hypoglycemia (times per & $9.25(2.9)$ & $7.4(7)$ & $7.0(5.63)$ \\
month) & & & \\
DQOL & $106.20(22.93)$ & $107.55(18.60)$ & $94.60(13.8 I)$ \\
DTSQ & $57.65(11.17)$ & $57.70(7.03)$ & $62.75(6.21)$ \\
CHFS-II & $35.55(12.24)$ & $42.25(18.27)$ & $32.25(11.84)$ \\
\hline
\end{tabular}

Notes: Data are expressed as mean (SD). Group A: SMBG group; Group B: FGM group; Group C: FGM combined WeChat-interactive management group.

Abbreviations: BMI, body mass index; LDL-C, low-density lipoprotein cholesterol; HDL-C, high-density lipoprotein cholesterol; TIR, time in range; TAR, time above range; TBR, time below range.

\section{Changes in Episodes of Hypoglycemia}

After the 6-month intervention, the number of hypoglycemic episodes per month in groups $\mathrm{B}$ and $\mathrm{C}$ decreased significantly from the baseline: Group A: $8.7 \pm 5.12$ vs $9.25 \pm 2.9$, respectively ( $p=0.324)$; Group B: $5.7 \pm 5.9$ vs $7.4 \pm 7$, respectively ( $p=0.03$ ); and Group C: $4.1 \pm 3.46$ vs $7.0 \pm 5.63$, respectively $(p<0.001)$. However, the magnitude of the decrease was higher in Group $\mathrm{C}$ than that observed in Group B $(p=0.02)$ (Figure 4).

\section{Changes in Self-Monitoring Level, Life Quality and Satisfaction Rate}

At the 6-month follow-up, the DMTSQ scores of all three groups increased from the baseline as follows: Group A: $57.65 \pm 11.17$ vs $56.70 \pm 10.94$, respectively $(p=0.03)$; Group B: $57.70 \pm 7.03$ vs $53.90 \pm 7.57$, respectively $(p<0.001)$; and Group C $62.75 \pm 6.21$ vs $56.00 \pm 5.88$, respectively $(p<0.001)$; the most significant increase was noted in Group C (Group A vs Group B, $p=0.003$; Group A vs Group C, $p<0.001$; Group B vs Group C, $p=0.006$ )

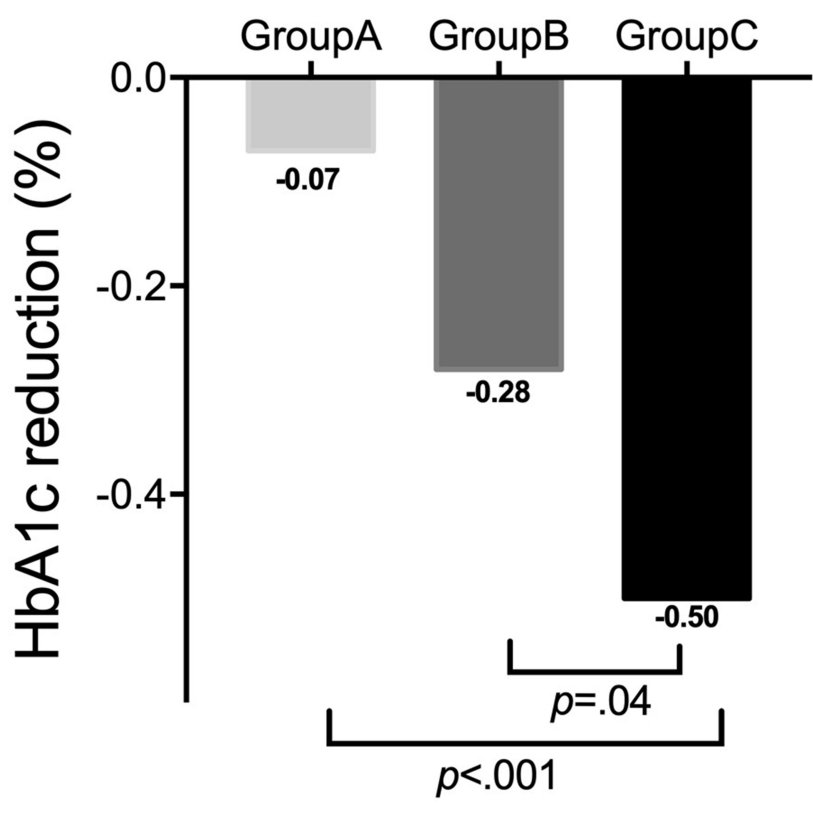

Figure 3 Comparison of $\mathrm{HbAlc}$ decline from baseline to 6 months later among SMBG, CGM, and CGM + WeChat groups of TID patients.

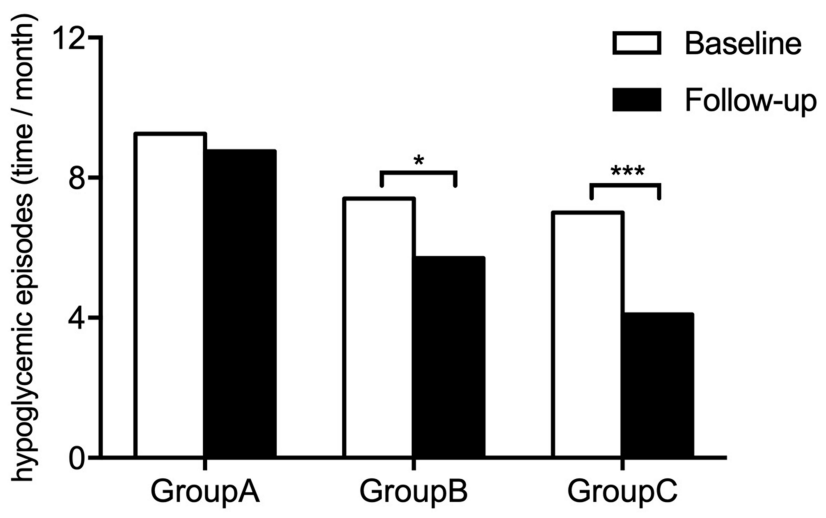

Figure 4 Incidence of hypoglycemia per month in three management groups. Notes: $* P<0.05$, $* * * P<0.001$ 6-month follow-up vs baseline.

(Figure 5A). Similarly, the DQOL scores in groups B and $\mathrm{C}$ decreased from the baseline, whereas the scores of Group A did not change significantly: Group A: $106.20 \pm 22.93$ vs $106.35 \pm 22.16$, respectively $(p=0.75)$; Group B: $108.5 \pm 17.75$ vs $111.20 \pm 16.36$, respectively $(p=0.001)$; and Group C: $93.95 \pm 14.57$ vs $100.95 \pm 17.96$, respectively $(p=0.002)$. The decrease in Group $\mathrm{C}$ was greater than that observed in Group B $(p=0.048)$ (Figure 5B). The CHFSII scores in all three groups at the 6th month follow-up decreased from the baseline, as follows: Group A: $31.85 \pm 11.59$ vs $35.55 \pm 12.24$, respectively ( $p<0.001$ ); Group B: $37.30 \pm 16.66$ vs $42.25 \pm 18.27$, respectively ( $p<0.001)$; and Group C: $24.6 \pm 10.67$ vs $32.25 \pm$ 11.84, respectively ( $p<0.001$ ), with significant intergroup differences in Group A vs Group C $(p<0.001)$ and Group 


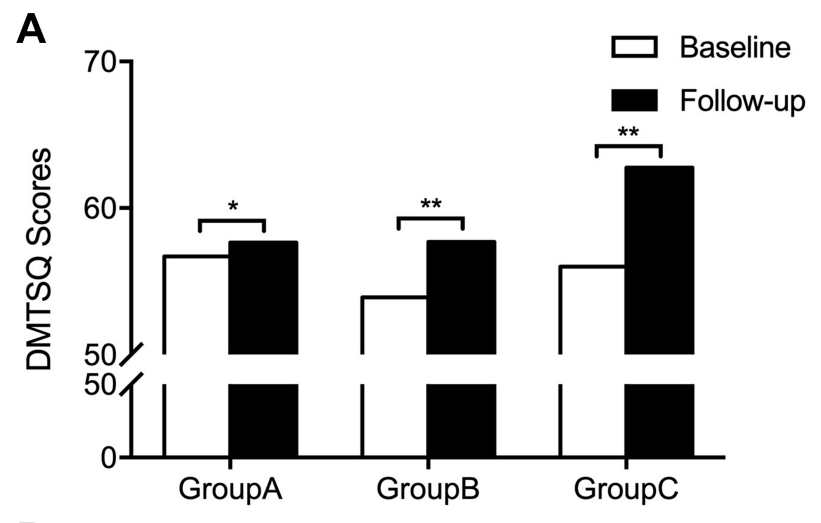

\section{B}
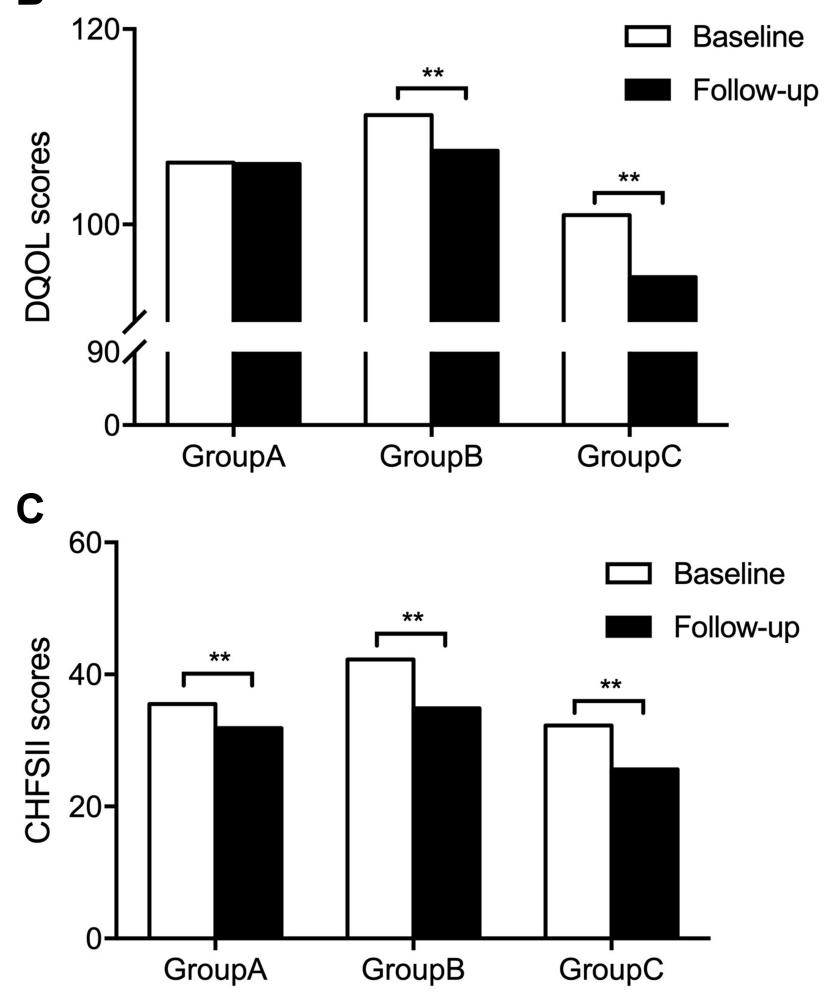

Figure 5 Effects of SMBG, FGM, and FGM combined WeChat-interactive management methods on quality of life and self-perception of TID patients. (A) Changes of DMTSQ Scores in three groups. (B) Changes of DQOL Scores in three groups. (C) Changes of CHFSII Scores in three groups.

Notes: $* P<0.05, * * P<0.016$ months later vs baseline.

B vs Group C ( $p=0.018$ ) (Figure 5C). And the comparison of the fluctuation range of each indicator is shown in the Supplementary Figure 1.

\section{Discussion}

In this study, we found that the $\mathrm{HbAlc}$ levels and the number of hypoglycemic episodes per month decreased from the baseline in all three groups after 6 months of intervention, with the greatest decrease observed in the FGM combined WeChat-interactive management group. The FGM groups
(Group B and Group C) showed a greater improvement in DQOL scores. Therefore, our novel integrated WeChat-Flash CGM model achieved a convenient doctor-patient interaction and further optimized the management effects.

The incidence of type 1 diabetes under 15 years old increased rapidly in the past two decades in China. ${ }^{1}$ Chinese adolescents with type 1 diabetes struggle with considerable challenges, including lower selfmanagement, high depressive symptoms, suboptimal metabolic control, and quality of life. ${ }^{13}$

Retrospective studies demonstrated that CGM achieved great glycemic benefit in pediatric, adolescent patients. ${ }^{14,15}$ As CGM becomes more routine, especially given the potential benefit of early CGM initiation, ${ }^{16}$ the decision-making process about initiating CGM may be less tangled for patients and families.

Recent studies have shown that smartphone-based apps are an efficient and effective tool for diabetes management. ${ }^{17-19}$ Kirwan et al used an app called Glucose Buddy in a 9-month follow-up randomized study, which enrolled 72 patients with T1D, and found that $\mathrm{HbA} 1 \mathrm{c}$ decreased by an average of $1.28 \%$ (from 9.08\% [SD 1.18] to 7.8\% [SD 0.75]) in the intervention group and an average of $0.11 \%$ (from $8.47 \%$ [SD 0.86] to $8.58 \%$ [SD 1.16]) in the control group. ${ }^{20}$ In their randomized clinical trial, Zhang et al concluded that the app interactive management group showed a greater reduction in $\mathrm{HbA} 1 \mathrm{c}(2.03 \%$ vs $1.37 \%)$ when compared to studies with the control group. ${ }^{21}$ Consistent with this previous study, our trial showed a greater improvement in $\mathrm{HbAlc}$ level in the WeChat interactive group after a 6-month follow-up. However, the improvement in HbA1c levels identified in this study was not as high as that observed in previous studies. ${ }^{20,21}$ This may have been due to a much lower baseline HbAlc level in our study compared to that observed for the other studies.

Improving quality of life (QOL) for children and their families while maintaining glycemic control within targets is a substantial challenge in diabetes treatment. ${ }^{22}$ A previous study showed that CGM could positively influence glucose control and help adolescent patients with T1D to enhance their quality of life. ${ }^{23}$ DQOL domains usually refer to treatment satisfaction, family relationships, self-efficacy, lifestyle flexibility, anxiety, fear of glucose fluctuations, fear of diabetes complications, and treatment expectations. ${ }^{24,25}$ Hence, apart from the HbAlc level, our study included QOL as a second major outcome to ensure 
that developing technologies have a positive impact on children and families with diabetes.

The design of this study was adjusted based on previous recent study outcomes on this topic. Zhang et al conducted a national web-based survey on use, perspectives, and attitudes regarding diabetes management mobile apps among patients with diabetes and diabetologists in China. ${ }^{26}$ They found that diabetes education, knowledge, patient-doctor communication, and diabetes diaries were considered the most important functions of a diabetes app. Performance expectancy and social influence are the most important determinants of the intention to use diabetes management apps. ${ }^{27}$ Thus, our WeChat public account platform had these elements integrated into each module. We observed that sending diabetic health education messages via the WeChat platform to diabetes patients was regarded as the most useful module through the endpoint interview, which supports that self-care behaviors (such as appropriate diet, regular exercise, and medication) contributed to achieving effective diabetes control.

In this study, we optimized the method for blood glucose monitoring in adolescents with T1D and used the internet to improve the conventional diabetes education model to strengthen the patient's understanding of T1D. Specifically, the flash glucose monitoring system used in this study was able to record blood glucose values at any time and allowed adolescent patients to receive warnings that indicated hypoglycemia or hyperglycemia. Accordingly, the patients were able to make adjustments to their diet and medication. Furthermore, the WeChat platform is a widely used social software app in China. Patients do not have to download, install, and register on other apps since WeChat has multipurpose features. Furthermore, it could make patients receive professional suggestions from the physician online and save the roundtrip time. It can help juvenile patients gain professional knowledge of diabetes, strengthen self-management consciousness, reduce the fear of the disease, and change unhealthy habits. There have been no similar research studies in China; thus, this presents preliminary insights into a novel glycemic monitoring method.

The study has some limitations. First, the sample size of the present study was small, and further studies are needed to recruit more participants. Second, this was a single-center study with a follow-up time of only 6 months. Considering the chronic characteristics of diabetes, the study could not assess the long-term impact of FGM combined WeChat-interactive model on diabetes management. Third, though statistically non-significant, there were differences in diabetes duration by 1 year between Groups A, B, and C. It is well known that most people with T1D have a honeymoon period of 6 months after diagnosis, which can last 1-2 years. So, this difference in diabetes duration may have affected the outcome. Finally, owing to the trial being an internet-based intervention, this study required high self-consciousness.

\section{Conclusion}

Our study suggests that the FGM combined with the WeChat-based support model can improve glycemic control, reduce the incidence of hypoglycemia, improve blood glucose fluctuations, and increase diabetes-related awareness in adolescents patients with T1D. It may be another solid clinical evidence for the feasibility and effectiveness of digital diabetes. Therefore, it might be popularized and implemented in the management of T1D, especially in the context of the current COVID-19 pandemic.

\section{Abbreviations}

CGM, continuous glucose monitoring systems; DCCT, Diabetes Control and Complications Trial; DMTSQ, Diabetes Monitoring and Treatment Satisfaction Questionnaire; DQOL, Diabetes Specific Quality of Life; FGM, flash glucose monitoring system; FPG, fasting plasma glucose; HbAlc, glycated hemoglobin Alc; ISFG, interstitial fluid glucose concentrations; QOL, quality of life; SMBG, self-monitoring of blood glucose; T1D, type 1 diabetes mellitus; CSII, continuous subcutaneous insulin infusion; MDI, multiple daily insulin.

\section{Data Sharing Statement}

After publication, the authors intend to share individual deidentified participant data, for 1 year, when asked by e-mail from the corresponding author upon reasonable request.

\section{Acknowledgments}

The authors would like to thank all volunteers who participated in this study for their dedication to data collection and laboratory measurements. The authors are also very grateful to all staff of the Department of Endocrinology and Metabolism of Shanghai Jiao Tong University Affiliated Sixth People's Hospital for trial implementation.

\section{Author Contributions}

All authors made a significant contribution to the work reported, whether that is in the conception, study design, 
execution, acquisition of data, analysis and interpretation, or in all these areas; took part in drafting, revising, or critically reviewing the article; gave final approval of the version to be published; have agreed on the journal to which the article has been submitted; and agree to be accountable for all aspects of the work.

\section{Funding}

This research was supported by grants from the National key Research and development program (2017YFC1309601 for Fang Liu), National Science Foundation Items of China (81770802 for Fang Liu), and Shanghai Municipal Education Commission-Gaofeng Clinical Medicine (20152232 for Fang Liu).

\section{Disclosure}

The authors report no conflicts of interest for this work.

\section{References}

1. Weng J, Zhou Z, Guo L, et al. Incidence of type 1 diabetes in China, 2010-13: population based study. BMJ. 2018;360:j5295. doi:10.1136/ bmj.j5295

2. Nwokolo M, Amiel SA, O'Daly O, et al. Impaired awareness of hypoglycemia disrupts blood flow to brain regions involved in arousal and decision making in type 1 diabetes. Diabetes Care. 2019;42 (11):2127-2135. doi:10.2337/dc19-0337

3. Umpierrez G, Korytkowski M. Diabetic emergencies - ketoacidosis, hyperglycaemic hyperosmolar state and hypoglycaemia. Nat Rev Endocrinol. 2016;12(4):222-232. doi:10.1038/nrendo.2016.15

4. Ziegler R, Heidtmann B, Hilgard D, Hofer S, Rosenbauer J, Holl R. Frequency of SMBG correlates with $\mathrm{HbAlc}$ and acute complications in children and adolescents with type 1 diabetes. Pediatr Diabetes. 2011;12(1):11-17. doi:10.1111/j.1399-5448.2010.00650.x

5. Maia FFR, Araújo LR. Efficacy of continuous glucose monitoring system (CGMS) to detect postprandial hyperglycemia and unrecognized hypoglycemia in type 1 diabetic patients. Diabetes Res Clin Pract. 2007;75(1):30-34. doi:10.1016/j.diabres.2006.05.009

6. Boland E, Monsod T, Delucia M, Brandt CA, Fernando S, Tamborlane WV. Limitations of conventional methods of self-monitoring of blood glucose: lessons learned from 3 days of continuous glucose sensing in pediatric patients with type 1 diabetes. Diabetes Care. 2001;24(11):1858-1862. doi:10.2337/ diacare.24.11.1858

7. Fakih El Khoury C, Karavetian M, Halfens RJG, Crutzen R, Khoja L, Schols JMGA. The effects of dietary mobile apps on nutritional outcomes in adults with chronic diseases: a systematic review and meta-analysis. J Acad Nutr Diet. 2019;119(4):626-651. doi:10.1016/ j.jand.2018.11.010

8. Eng DS, Lee JM. The promise and peril of mobile health applications for diabetes and endocrinology. Pediatr Diabetes. 2013;14 (4):231-238. doi:10.1111/pedi.12034

9. El-Gayar O, Timsina P, Nawar N, Eid W. Mobile applications for diabetes self-management: status and potential. J Diabetes Sci Technol. 2013;7(1):247-262. doi:10.1177/193229681300700130

10. Chomutare T, Fernandez-Luque L, Arsand E, Hartvigsen G. Features of mobile diabetes applications: review of the literature and analysis of current applications compared against evidence-based guidelines. J Med Internet Res. 2011;13(3):e65. doi:10.2196/jmir.1874
11. Zhang X, Wen D, Liang J, Lei J. How the public uses social media wechat to obtain health information in china: a survey study. $B M C$ Med Inform Decis Mak. 2017;17(Suppl 2):66. doi:10.1186/s12911017-0470-0

12. He C, Wu S, Zhao Y, et al. Social media-promoted weight loss among an occupational population: cohort study using a WeChat mobile phone app-based campaign. J Med Internet Res. 2017;19(10):e357. doi:10.2196/jmir.7861

13. Guo J, Whittemore R, Grey M, Wang J, Zhou ZG, He GP. Diabetes self-management, depressive symptoms, quality of life and metabolic control in youth with type 1 diabetes in China. J Clin Nurs. 2013;22 (1-2):69-79. doi:10.1111/j.1365-2702.2012.04299.x

14. Miller KM, Foster NC, Beck RW, et al. Current state of type 1 diabetes treatment in the U.S.: updated data from the T1D exchange clinic registry. Diabetes Care. 2015;38(6):971-978. doi:10.2337/ dc15-0078

15. Beck RW, Riddlesworth T, Ruedy K, et al. Effect of continuous glucose monitoring on glycemic control in adults with type 1 diabetes using insulin injections: the DIAMOND randomized clinical trial. JAMA. 2017;317(4):371-378. doi:10.1001/jama.2016.19975

16. Mulinacci G, Alonso GT, Snell-Bergeon JK, Shah VN. Glycemic outcomes with early initiation of continuous glucose monitoring system in recently diagnosed patients with type 1 diabetes. Diabetes Technol Ther. 2019;21(1):6-10. doi:10.1089/dia.2018.0257

17. Hou C, Carter B, Hewitt J, Francisa T, Mayor S. Do mobile phone applications improve glycemic control (HbAlc) in the self-management of diabetes? A systematic review, meta-analysis, and GRADE of 14 randomized trials. Diabetes Care. 2016;39 (11):2089-2095. doi:10.2337/dc16-0346

18. Quevedo Rodríguez A, Wägner AM. Mobile phone applications for diabetes management: a systematic review. Endocrinol Diabetes Nutr. 2019;66(5):330-337. doi:10.1016/j.endinu.2018.11.005

19. Gunawardena KC, Jackson R, Robinett I, et al. The influence of the smart glucose manager mobile application on diabetes management. J Diabetes Sci Technol. 2019;13(1):75-81. doi:10.1177/193229 6818804522

20. Kirwan M, Vandelanotte C, Fenning A, Duncan MJ. Diabetes self-management smartphone application for adults with type 1 diabetes: randomized controlled trial. J Med Internet Res. 2013;15(11): e235. doi:10.2196/jmir.2588

21. Zhang L, He X, Shen Y, et al. Effectiveness of smartphone app-based interactive management on glycemic control in chinese patients with poorly controlled diabetes: randomized controlled trial. J Med Internet Res. 2019;21(12):e15401. doi:10.2196/15401

22. Hirose M, Beverly EA, Weinger K. Quality of life and technology: impact on children and families with diabetes. Curr Diab Rep. 2012;12(6):711-720. doi:10.1007/s11892-012-0313-4

23. Polonsky WH, Hessler D, Ruedy KJ, Beck RW. The impact of continuous glucose monitoring on markers of quality of life in adults with type 1 diabetes: further findings from the DIAMOND randomized clinical trial. Diabetes Care. 2017;40(6):736-741. doi: $10.2337 / \mathrm{dc} 17-0133$

24. Peyrot M. The DAWN youth WebTalk study: methods, findings, and implications. Pediatr Diabetes. 2009;10(Suppl 13):37-45. doi:10.1111/j.1399-5448.2009.00612.x

25. Wallander JL, Schmitt M, Koot HM. Quality of life measurement in children and adolescents: issues, instruments, and applications. J Clin Psychol. 2001;57(4):571-585. doi:10.1002/jclp.1029

26. Zhang Y, Li X, Luo S, et al. Use, perspectives, and attitudes regarding diabetes management mobile apps among diabetes patients and diabetologists in China: national web-based survey. JMIR mHealth uHealth. 2019;7(2):e12658. doi:10.2196/12658

27. Zhang Y, Liu C, Luo S, et al. Factors influencing patients' intentions to use diabetes management apps based on an extended unified theory of acceptance and use of technology model: web-based survey. $J$ Med Internet Res. 2019;21(8):e15023. doi:10.2196/15023 


\section{Publish your work in this journal}

Diabetes, Metabolic Syndrome and Obesity: Targets and Therapy is an international, peer-reviewed open-access journal committed to the rapid publication of the latest laboratory and clinical findings in the fields of diabetes, metabolic syndrome and obesity research. Original research, review, case reports, hypothesis formation, expert opinion and commentaries are all considered for publication. The manuscript management system is completely online and includes a very quick and fair peer-review system, which is all easy to use. Visit http://www.dovepress.com/testimonials.php to read real quotes from published authors. 